\title{
Upregulation of P2Y12 inhibits chondrocyte apoptosis in lumbar osteoarthritis through PI3K/AKT signaling pathway
}

\section{Guanyin Wu ( $16621370855 @ 163 . c o m)$}

Nantong City No 1 People's Hospital and Second Affiliated Hospital of Nantong University https://orcid.org/0000-0001-9305-8970

\section{Pengfei Xue}

Nantong City No 1 People's Hospital and Second Affiliated Hospital of Nantong University

Hurich Jin

Nantong City No 1 People's Hospital and Second Affiliated Hospital of Nantong University

\section{Mo Zhang}

Nantong City No 1 People's Hospital and Second Affiliated Hospital of Nantong University

Chao Gui

Nantong City No 1 People's Hospital and Second Affiliated Hospital of Nantong University

\section{Guofeng Bao}

Nantong City No 1 People's Hospital and Second Affiliated Hospital of Nantong University

\section{Guanhua Xu}

Nantong City No 1 People's Hospital and Second Affiliated Hospital of Nantong University

Jiawei Jiang

Nantong City No 1 People's Hospital and Second Affiliated Hospital of Nantong University Jinlong Zhang

Nantong City No 1 People's Hospital and Second Affiliated Hospital of Nantong University Shengyu Cui

Nantong City No 1 People's Hospital and Second Affiliated Hospital of Nantong University Zhiming Cui

Nantong City No 1 People's Hospital and Second Affiliated Hospital of Nantong University Yuyu Sun

Affiliated Nantong Hospital 3 of Nantong University

\section{Research Article}

Keywords: P2Y12, FJOA, Osteoarthritis, Apoptosis,PI3K/Akt.

Posted Date: February 24th, 2022 
DOI: https://doi.org/10.21203/rs.3.rs-1099620/v2

License: (c) (1) This work is licensed under a Creative Commons Attribution 4.0 International License. Read Full License 


\section{Abstract}

\section{Background}

Lumbar facet osteoarthritis (FJOA) is one of the major causes of severe low back pain and disability worldwide. However, the underlying mechanism of cartilage degeneration in FJOA remains unclear.

\section{Methods}

The rats were randomly divided into non-operation group $(n=20)$ and operation group $(n=10)$. In the operation group, MIA (Sigma, $200 \mathrm{mg} / \mathrm{mL}$ ) was injected into the right $\mathrm{L} 4 / 5$ facet process by blunt nanoneedle 26 (WPI, Sarasota, FL, USA) under the control of injection pump. The final injection volume was $5 \mu \mathrm{L}$ and the injection rate was $2 \mu \mathrm{L} / \mathrm{min}$. The facet joint was removed 4 weeks after surgery. After the operation, some samples were stored at $80^{\circ} \mathrm{C}$ until use. The right facet joints in each group were tested as follows. HE staining and iron red solid green staining were used to observe the degeneration of articular chondrocytes in rats. Immunohistochemistry and Western blot were used to observe the expression of P2Y12, MMP13, COL2 and other cartilage degeneration and apoptosis related indexes. Co-localization of P2Y12-Cleaved caspase-3 in apoptosis models was detected by dual-standard immunofluorescence staining. Apoptosis was detected by flow cytometry and TUNEL.

\section{Results}

The expression of P2Y12 increased in OA cartilage tissue, and P2Y12 inhibited apoptosis. Knockdown of P2Y12 promotes IL-1 $\beta$-induced apoptosis.

\section{Conclusion}

Our data suggest that P2Y12 has a chondroprotective effect on FJOA.

\section{Introduction}

Facet joints exhibit the typical characteristics of synovial joints: articular cartilage covers the opposite side of each facet joint, located on the thickened layer of subchondral bone, and the synovial membrane connects to the edge of the articular cartilage portion [1]. It constitutes three connected complexes of functional units [2]. Lumbar facet osteoarthritis (LFJOA) is one of the most common degenerative diseases of the spine. Facet joint degeneration (FJD) is the pathological basis of lumbar degeneration. Chronic low back pain can be caused by degenerative diseases, which is more common in middle-aged and elderly people [3]. The basic pathological features are articular chondrocyte damage, subchondral bone and periarticular bone hyperplasia and osteophyte formation of lumbar facet joints [4]. The occurrence of osteoarthritis (OA) is affected by many inflammatory factors, such as tumor necrosis factor a (TNF-A) and interleukin 1B (IL-1B) [5]. During the progression of FJOA, these pro-inflammatory cytokines are considered to be key factors in promoting chondrocyte apoptosis. In addition, activation of Caspase-3 has been proved to be involved in the occurrence of OA [6]. Caspase-3 is one of the most 
critical effector proteases in cell apoptosis. However, basic research on the evaluation of FJOA and its specific molecular mechanisms has been lacking for many years. P2Y12R gene is located on chromosome 3Q21-Q25 [7]. P2Y12 receptor (P2YR) is a G-coupled7-membrane-spanning proteins. They are activated by nucleotides and extracellular signaling molecules released by damaged cells under inflammatory, ischemic, and hypoxia conditions [8]. Activation of P2Y12 receptor first phosphorylates BAD through the PI3K Akt pathway, and the liquid acidified BAD binds to 14-3-3 protein to separate BAD from $B A D$ Bcl-XL complex, thus releasing free $B c l-X L$. Free $B c l-X L$ directly binds to $B A K$, downregulates the dimerization level of BAK and inhibits the activation of BAK. Although Bcl-XL and Bax do not bind directly in platelets, they may inhibit Bax activation by reducing the translocation of Bax from cytoplasm to mitochondria through some indirect effects. In nucleated cells, BAD is an important target gene downstream of Akt [9] and belongs to the pro-apoptotic Bcl-2 family of proteins. The pro-apoptotic function of bcl-XL is realized by binding to anti-apoptotic protein $\mathrm{Bcl}-\mathrm{XL}$ and inhibiting its anti-apoptotic activity. In nucleated cells, Akt activation inhibits their pro-apoptotic activity through flow acidification. As an important anti-apoptotic protein, dissociated Bcl-XL mainly binds to BAK or Bax and inhibits the porepromoting activity of BAK or Bax [10] to achieve its anti-apoptotic function. These findings suggest that $\mathrm{P} 2 \mathrm{Y} 12$ has the potential to regulate cell death, but its expression in lumbar facet joint osteoarthritis and its possible role remain unclear. These findings suggest that $\mathrm{P} 2 \mathrm{Y} 12$ has the potential to regulate cell death. Considering the similar phenotypes between SW1353 cells and human chondrocytes, which have been successfully applied in previous scientific experiments, we decided to use the SW1353 cell line for a series of experiments [11]. To our knowledge, this is the first study to evaluate differential expression, locate and explore the role of P2Y12 in FJOA. This study may provide useful clues and potential therapeutic targets for LFJ degeneration and arthritis induced low back pain.

\section{Materials And Methods}

\section{Experimental animals}

All animal experiments are in line with the National Health Center "Experimental Animal Nursing and Use Guide" and approved the Jiangsu Branch of China's National Medical Experimental Animal Committee. Male SD rats $(n=40)$, aged 40-50 days, weighing 200-250 g, are placed in a constant temperature room $\left(37 \pm 0.5^{\circ} \mathrm{C}\right)$, and light-dark cycle is 12 hours. Using the previously reported[12] intra-articular injection MIA modeling method, the surgical FJOA model was established in the facet joint. The rats were randomly divided into non-surgical group $(n=10)$ and surgical group $(n=10)$. The surgical group is under the control of the syringe pump, using a 26-gauge blunt nano fil needle (WPI, Sarasota, FL, USA) to inject MIA (Sigma, $200 \mathrm{mg} / \mathrm{mL}$ ) into the right $\mathrm{L} 4 / 5$ facet joint Inside, the final injection volume is $5 \mu \mathrm{L}$, and the injection rate is $2 \mu \mathrm{L} / \mathrm{min}$. The facet joints were collected 4 weeks after the operation. After the operation, some samples were stored at $-80^{\circ} \mathrm{C}$ until use. The remaining samples were fixed with $10 \%$ neutral buffered formalin, decalcified with $10 \%$ formic acid, and embedded in paraffin. Take the sagittal histological section of the articular chondrocytes of the right facet joints. The right facet joints of each group underwent following experiments. 


\section{Histopathologic Analysis}

The lumbar facet joints of rats were prepared and fixed with $4 \%$ paraformaldehyde. The sample was then decalcified in $10 \%$ EDTA for 21 days and embedded in paraffin. Tissue sections $(5 \mu \mathrm{m})$ were stained with Tibetan red $\mathrm{O} /$ solid green according to the standard regimen to determine cartilage degradation under light microscopy. The International Society for the Study of Osteoarthritis (OARSI) scoring system was used to evaluate articular cartilage degeneration [13]. Immunohistochemistry was further performed to analyze the protein expression of P2Y12, MMP13 and COL2 in the lumbar facet joint tissue sections of rats. Primary antibodies against P2Y12 (abcam), MMP13, and COL2 (proteintech) were used in 1:100-1 1:200 diluents and cultured overnight at $4^{\circ} \mathrm{C}$. The sections were then cultured with a 3-oxide drug and cellular longevity biotinylated secondary antibodies. DAB kits were used for reaction. The expression of P2Y12 and MMP13 was assessed by calculating the percentage of immune positive cells. COL2 expression was evaluated by calculating relative strength.

\section{Cell culture and stimulation}

Human SW1353 chondrosarcoma cells (Institute of Biochemistry and Cell Biology, Shanghai, China) were cultured in Lebovitz's L-15 medium, supplemented with $10 \%$ fetal bovine serum, $37^{\circ} \mathrm{C}$, and $5 \% \mathrm{CO} 2$ humidified air. The cells were passaged every 3 to 4 days. In the stimulation experiment, $20 \mathrm{ng} / \mathrm{ml}$ human IL-1 $\beta$ (proteintech) was added to the cells $0,6,12,24$, or 48 hours before harvesting.

\section{SiRNAs transfection}

P2Y12-siRNA comes from Guangzhou RiboBio Co. Ltd. The siRNA targeting the P2Y12 sequence is: 5'TGGGACTGATAACTATCGA-3'. Follow the manufacturer's instructions to transfect SW1353 cells with P2Y12-siRNA. After $48 \mathrm{~h}$ of transfection, the transfected cells were collected for subsequent experiments.

\section{Western blot analysis}

After the necessary treatments, the protein samples were collected from SW1353 cells with cell lysis buffer. $50 \mathrm{mg}$ of total protein was loaded into $10 \%$ sodium dodecyl sulfate polyacrylamide gel electrophoresis (SDS-PAGE) and printed on polyvinylidene difluoride (PVDF) membrane. Seal the membrane with $5 \%$ skimmed milk at room temperature for $1 \mathrm{~h}$, and use the primary antibody P2Y12 (antirabbit 1:500; proteintech). cleaved caspase-3 (anti-mouse, 1:1000, proteintech) MMP13 (anti-rabbit, 1:1000, proteintech) GAPDH (anti-rabbit, 1:1000, abcam); 4 degrees Celsius at night. After incubation with the secondary antibody conjugated with horseradish peroxidase (HRP), the protein was visualized using an enhanced chemiluminescence kit (ECL, Pierce).

\section{Double immunofluorescent staining}

Place the cells on a 24-well plate ( $2 \times 104$ cells/cover glass) and treat them with IL-1 $\beta(20 \mathrm{ng} / \mathrm{ml})$ for 24 hours. Then use P2Y12, cleaved caspase 3 primary antibody and fluorescently labeled secondary antibody to detect the localization of P2Y12 and cleaved caspase-3 in SW1353 cells. Finally, observe the cells with a Zeiss fluorescence microscope (Germany) or Leica confocal microscope (Germany). 


\section{Measurement of Apoptosis}

According to the manufacturer's instructions (Becton Dickinson), cells were stained in the dark with annexin V-FITC/ propidium iodide (PI) for $15 \mathrm{~min}$. The stained cells were detected by flow cytometry and analyzed by FlowJo software. Apoptosis of chondrocytes was detected using the Terminal deoxynucleotide transferase dUTP Notched-end Labeling (TUNEL) assay kit (Beyotime) according to manufacturer's instructions.

\section{Statistical analysis}

All data were analyzed using GraphPad Prism 10 software (GraphPad software, Inc., La Jolla, CA, USA). One-way analysis of variance, Tukey's post hoc multiple comparison test and double comparison unpaired $t$ test were used for statistical analysis. Expressed as $P<0.05$, which is statistically significant.

\section{Results}

\section{P2Y12 expression is up-regulated in the articular cartilage of rat lumbar facet joints}

In the FJOA model, articular chondrocyte P2Y12 expression was increased. It is doubtful whether P2Y12 participates in the FJOA process of FJOA model. Experimental FJOA model of SD rats injected with MIA was established to reveal the relationship between chondrocyte apoptosis and P2Y12. The changes of articular facet chondrocytes in the operation group and the non-operation group were reflected by the saffron solid green staining and H\&E staining. Compared with the operation group, the facet joints of the rats in the non-operation group were smooth and chondrocytes arranged more orderly (Figure 1A -B). OARSI grade scores confirmed cartilage damage caused by MIA injection (Figure 1C). Immunohistochemical analysis showed that compared with the non-surgical group, P2Y12 positive cells, MMP13 positive cells and COL2 positive cells were increased in the surgical group (Figure 1D). Western blot analysis also confirmed that the expression of P2Y12 was consistent with markers of facet joint degeneration (Figure 1E and F).

\section{IL-1 $\beta$ induced increase in P2Y12 expression and apoptosis in SW1353 cells}

Considering that the main pathogenic mechanism of FJOA is caspase-dependent apoptosis in chondrocytes, an IL-1 $\beta$ induced SW1353 chondrocyte apoptosis model was established, revealing the relationship between chondrocyte apoptosis and P2Y12. Western blot analysis showed the temporal expression pattern of P2Y12 in the chondrocyte apoptosis model. As shown in A and B in Figure 2, it was confirmed that the expression of P2Y12 was low at 0 hours, but after IL-1 $\beta$ induction, P2Y12 was upregulated at 12 hours and reached a peak at 36 hours $(P<0.05)$.Immune blot analysis is also used to discover the expression of the known apoptosis marker caspase- 3 and Bax. In the IL-1 $\beta$ induced SW1353 cell apoptosis model, the levels of cleaved caspase- 3 and Bax were up-regulated at 12 hours and reached 
a peak at 36 hours. In addition, cell immunofluorescence revealed the co-localization of P2Y12 and cleaved caspase-3 under IL-1ßstimulation, as shown in Figure $2 \mathrm{C}$. These data indicate that during the progression of FJOA, the caspase-dependent apoptotic pathway may play an important role between P2Y12 and chondrocyte apoptosis.

\section{Inhibition of P2Y12 promote IL-1 $\beta$ to induce SW1353 cell apoptosis}

To further determine the correlation between P2Y12 and chondrocyte apoptosis, SW1353 cells transfected with siRNA were used to knock down the expression of P2Y12 protein. 48 hours after transfection, P2Y12-siRNA, non-specific siRNA and negative control were detected. As shown in Figure 3A and B, in the IL-1ßinduced SW1353 cell model, siRNA significantly inhibited P2Y12 expression. In order to identify the relationship between $\mathrm{P} 2 \mathrm{Y} 12$ and cell apoptosis, we observed the increased expression of caspase-3 and Bax protein activated after P2Y12 silence in the SW1353 cell apoptosis model (Figure 3C and D). These data indicate that P2Y12 may inhibit the expression of caspase-3 and Bax in chondrocytes in FJOA.

\section{P2Y12 negatively regulates the apoptosis of SW1353 cells induced by IL-1 $\beta$}

As our above findings indicate that P2Y12 is closely related to chondrocyte apoptosis in FJOA, it is necessary to further evaluate the effect of P2Y12 on IL-1 Binduced apoptosis of SW1353 cells through Annexin V/PI based on flow cytometry. dyeing. Compared with the control group, we found that the number of apoptotic cells in P2Y12-siRNA cells increased significantly (Figure 4). Taken together, these data indicate that P2Y12 may inhibit inflammation-induced chondrocyte apoptosis during FJOA.

\section{Effects of P2Y12 on PI3K/AKT signaling in IL-1ß-induced chondrocytes}

To investigate the mechanism by which inhibition of P2Y12 function in chondrocytes increases IL-1 $\beta$ induced chondrocyte apoptosis, we performed Western blot analysis of signaling molecules involved in the PI3K/AKT signaling pathway. We found that reduced P2Y12 expression in chondrocytes activates the $\mathrm{PI} 3 \mathrm{~K} / \mathrm{AKT}$ signaling pathway due to increased phosphorylation at Ser473, the active form of AKT. As shown in Figure 5A and 5B, after transfection with SiP2Y12, the phosphorylation level of AKT in IL-1 $\beta$ treated chondrocytes increased, but the total protein amount of AKT in treated cells did not change. To investigate whether the effect of P2Y12 on chondrocytes is modulated by the PI3K/ AKT signaling pathway, we treated chondrocytes with LY294002, a specific PI3K inhibitor, in the presence of IL-1 $\beta$. Western blot analysis showed that Cleaved Caspase 3 expression was decreased and BCL 2 expression was increased after LY294002 treatment of SiP2Y12 transfected chondrocytes (Figure 5C-5F).

Subsequently, TUNEL experiment was performed to further verify the above results (FIG. G)In conclusion, these results suggest that $\mathrm{PI} 3 \mathrm{~K} / \mathrm{AKT}$ signaling pathway is involved in the regulation of $\mathrm{P} 2 \mathrm{Y} 12$ on chondrocyte apoptosis 


\section{Discussion}

Although factors such as age, genetics, mechanical stress, trauma and metabolism are associated with OA progression, the cellular signaling and metabolic changes that occur in chondrocytes are not fully understood. In this study, we found that the expression of P2Y12 increased during the progression of OA, and $\mathrm{P} 2 \mathrm{Y} 12$ regulated apoptosis and ECM degradation by regulating the PI3K/AKT signaling pathway, thus changing the homeostasis of cartilage. In terms of mechanism, we found that P2Y12 inhibits the binding of Bad and Bcl2 through the Pi3k/Akt pathway, thus inhibiting apoptosis. Then, we further applied the OA animal model to verify the potential therapeutic effect of P2Y12 in the progression of OA. The P2Y receptor is a G-coupled transmembrane protein that exists in almost any cell type and ligands are purine and pyrimidine nucleotides. From the phylogenetic and structural perspective, two P2Y receptor subgroups with high structural differences were identified. The first subgroup includes $\mathrm{G}_{\mathrm{q}}$-coupled subgroups (P2Y1, P2Y2, P2Y4, P2Y6 and P2Y11). The second subgroup includes $G_{i}$ coupling subtypes (P2Y12, P2Y13, and P2Y14) [14]. P2y12-like receptors in apoptosis and inflammation have been reported to be involved in platelets [15]and microglia [16-18] chemotaxis and phagocytosis. Although facet arthritis and microglial inflammation share many similar features of joint injury, they represent different etiologies and pathologic processes. Whether $\mathrm{P} 2 \mathrm{Y} 12$ regulates Cartilage steady-state in the pathogenesis of OA has not been studied in detail. Our data suggest that P2Y12 expression level is associated with OA severity.

Considering the correlation between the expression of P2Y12 and OA conditions, we induced OA by injecting MIA to establish a rat articular process FJOA model, and MIA has been widely used to study the pathogenesis of OA [19-20]. The lumbar facet joints were then taken and divided into non-operative and operative groups. Immunohistochemical staining showed significantly increased P2Y12 expression in articular cartilage of severely degenerative FJOA. In vitro Western blot analysis showed that P2Y12 and Bax and Caspase-3 expression were significantly increased after $36 \mathrm{~h}$ of IL-1 $\beta$ stimulation on SW1353 cells. II- $1 \beta$ is a key pro-inflammatory cytokine in the pathogenesis of $O A$ and is usually used to stimulate chondrocytes to simulate OA conditions in vitro studies [21-23]. In addition, co-localization of P2Y12/ Cleaved caspase-3 in chondrocyte apoptosis models was detected by double-labeled immunofluorescence staining. SiRNA silencing P2Y12 increased il-1 $\beta$-induced Bax and Caspase-3 cleavage expression, suggesting that $\mathrm{P} 2 \mathrm{Y} 12$ negatively regulates chondrocyte apoptosis through caspase-dependent signaling pathways. Flow cytometry showed that inhibition of P2Y12 significantly enhanced IL-1 $\beta$-induced apoptosis of SW1353 cells. In order to investigate the mechanism of inhibiting chondrocyte P2Y12 function and increasing IL-1 $\beta$ induced chondrocyte apoptosis, we analyzed signaling molecules in the PI3K/AKT signaling pathway by Western blot. We treated chondrocytes with the specific PI3K inhibitor LY294002 in the presence of IL-1 3 . Western blot analysis showed that Cleaved Caspase 3 and Bcl2 expression were consistent with control group after siPP2Y12 transfected chondrocytes treated with LY294002. TUNEL staining analysis showed that the number of apoptotic cells was up-regulated after siPP2Y12 transfected with LY294002. Based on our data, we conclude that P2Y12 may play a key role in inhibiting the apoptosis of articular chondrocytes in rats. 
In the lumbar region, facet joints play the most important role in maintaining the stability of spinal movement. FJOA is a degenerative joint disease characterized by chondrocyte degeneration [24] and chondrocyte apoptosis [25]. However, the exact molecular mechanism of chondrocyte apoptosis remains unclear. In this study, we focused on the role of P2Y12 in chondrocyte apoptosis in lumbar facet joint osteoarthritis. First, we established a rat articular process FJOA model, and then took the lumbar facet joints and divided them into non-surgical and surgical group. Immunohistochemical staining showed that the expression of P2Y12 in the articular cartilage of severely degenerated FJOA was significantly increased. In vitro, Western blot analysis showed that P2Y12 was significantly increased $36 \mathrm{~h}$ after IL-1 $\beta$ stimulated SW1353 cells, and caspase-3 was also up-regulated. In addition, the co-localization of P2Y12/cleaved caspase-3 was detected in the cell chondrocyte apoptosis model by doubl-labeled immunofluorescence staining. Silencing of P2Y12 by siRNA can increase the expression of caspase-3 cleavage induced by IL-1 $\beta$, indicating that $\mathrm{P} 2 \mathrm{Y} 12$ negatively regulates chondrocyte apoptosis through a caspase-dependent signaling pathway. Flow cytometry showed that P2Y12 knockdown significantly enhanced IL-1 $\beta$ induced apoptosis of SW1353 cells. Based on our data, we conclude that P2Y12 may play a key role in inhibiting the apoptosis of rat articular chondrocytes through the PI3K/AKT signaling pathway.

As a G protein coupled receptor, P2Y12 has the typical characteristics of 7 hydrophobic transmembrane (TM) regions, which are connected by 3 extracellular loops (EL) and 3 intracellular loops [25]. The human $\mathrm{P} 2 \mathrm{Y} 12$ receptor consists of 342 amino acid residues [7]. Like most $\mathrm{G}$ protein coupled receptors, P2Y12 receptors are in the extracellular N-terminal (Cys17), the first extracellular ring (Cys97), the second extracellular ring (CYS175) and the third extracellular ring (Cys270) Has 4 extracellular hormone residues [16]. The P2Y12 receptor is coupled to the Gai2 subunit [26]. After stimulation, $\mathrm{Ga}$ and GßY subunits dissociate to activate various signal transduction pathways [27]. The G $\beta Y$ subunit stimulates the activity of phosphatidylinositol 3 kinase (PI3K), which leads to the late accumulation of phosphatidylinositol 3,4bisphosphate [Ptdlns $(3,4)$ P2] and phosphatidylinositol 3,4,5-Triphosphate [Ptdlns $(3,4)$ rapid transient accumulation, 5) P3] [27-29]. The PI3K pathway also activates Rap1b [9]and Akt [30]. Gßy dimers can activate inward rectifier potassium channels (GIRKs) that mediate Src tyrosine kinase G protein-gated inward rectifier potassium channels (GIRKs) [31]. The P2Y12 receptor inhibits apoptosis by activating the PI3K/Akt pathway: P2Y12 activation induces Akt and Bad phosphorylation. After being phosphorylated by Akt, Bad is sequestered in the cytoplasm by the adaptor protein 14-3-3, thereby preventing the binding of Bad to Bcl-XL. As a result, the free Bcl-xL heterodimer with Bak protein prevents the dimerization of Bak in the mitochondria, thereby antagonizing its pro-apoptotic activity. Bcl-xL indirectly inactivates Bax by inhibiting its translocation to mitochondria [10].

P2Y12 receptors are mainly expressed in platelets and neuronal tissues [31]. It is worth noting that few studies have reported the function of P2Y12 in the chondrocytes of the lumbar facet joints. In our research, we comprehensively show that $\mathrm{P} 2 \mathrm{Y} 12$ is up-regulated in cartilage and participates in the development of FJOA. In normal cartilage, the expression of P2Y12 is weak, while in the joint cartilage of the most severe surgical group, the expression of $\mathrm{P} 2 \mathrm{Y} 12$ is higher. A positive correlation was obtained 
between the expression of $\mathrm{P} 2 \mathrm{Y} 12$ and the severity of FJOA. Our hypothesis is that $\mathrm{P} 2 \mathrm{Y} 12$ plays an important role in FJOA and may be one of the key factors in inhibiting chondrocyte apoptosis.

\section{Conclusions}

In conclusion, $\mathrm{P} 2 \mathrm{Y} 12$ expression is significantly up-regulated in lumbar facet joint osteoarthritis. Inhibition of P2Y12 enhanced the expression of SW1353 cell apoptosis induced by IL-1 $\beta$. These observations indicate that the up-regulation of P2Y12 may play an important role in inhibiting FJOA chondrocyte apoptosis. Further research on the function of P2Y12 in chondrocytes may provide new insights into the pathophysiology of FJOA and advanced strategies for the treatment of FJOA.

\section{Abbreviations}

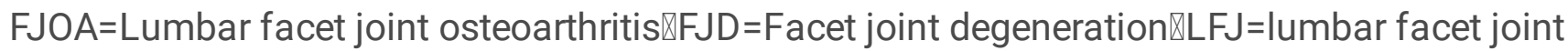

\section{Declarations}

\section{Acknowledgements}

This work was supported by Scientific research project of Nantong health and Family Planning Commission (QA2019013), Mandatory subject of Nantong Science and Technology Bureau(JC2020018)凹 Guiding projects of Nantong Municipal Science and technology plan囚JCZ20126『,Nantong Municipal Health Commission囚JCZ21019区.

\section{Author contributions}

Guanyin Wu, Pengfei Xue and Jinhuricha; Mo Zhang designed and supervised the study; Chao Gui,Guofeng Bao,Guanhua Xuand Jiawei Jiang performed the research; Jinlong Zhang andShengyu Cui analyzed the data; Yuyu Sun wrote the paper. All authors have read and approved submission of the final manuscript

\section{Funding}

This work was supported by Scientific research project of Nantong health and Family Planning Commission (QA2019013), Mandatory subject of Nantong Science and Technology Bureau(JC2020018)》 Guiding projects of Nantong Municipal Science and technology plan囚JCZ20126邓,Nantong Municipal Health Commission区JCZ21019区.

\section{Availability of data and materials}

All data generated or analyzed during this study are included in this 
published article.

\section{Compliance with ethical standards}

\section{Competing interests}

The authors have no competing interests to declare.

\section{Ethical approval}

Ethical approval was obtained from the Affiliated Hospital 2 of Nantong University Research Ethics Committee.

Consent to participate Not applicable.

Consent for publication Not applicable.

\section{References}

1. Gellhorn AC, Katz JN, Suri P (2013 Apr) Osteoarthritis of the spine: the facet joints. Nat Rev Rheumatol 9(4):216-224. doi: 10.1038/nrrheum.2012.199. Epub 2012 Nov 13. PMID: 23147891; PMCID: PMC4012322

2. Yong-Hing K, Kirkaldy-Willis WH The pathophysiology of degenerative disease of the lumbar spine.Orthop Clin North Am. 1983Jul; 14(3):491-504. PMID: 6346204.

3. Chu C, Chunshuai W, Jiajia C, Jinlong Z, Pengfei X, Jiawei J, Zhiming C Transcriptional information revealed differentially expressed circular RNAs in facet joint osteoarthritis. Biochem Biophys Res Commun. 2018 Mar 4;497(2):790-796. doi: 10.1016/j.bbrc.2018.02.157. Epub 2018 Feb 19. PMID: 29470979

4. Li J, Muehleman C, Abe Y, Masuda K Prevalence of facet joint degeneration in association with intervertebral joint degeneration in a sample of organ donors.J Orthop Res. 2011Aug; 29(8):126774. doi: 10.1002/jor.21387. Epub 2011 Feb 25. PMID: 21360583; PMCID: PMC3115475.

5. Lianxu C, Hongti J et al (2006) "NF-kappaBp65-specific siRNA inhibits expression of genes of COX-2, NOS-2 and MMP-9 in rat IL-1beta-induced and TNF-alpha-induced chondrocytes. " Osteoarthr Cartil 14(4):367-376

6. Ju XD, Deng M, Ao YF, Yu CL, Wang JQ, Yu JK, Cui GQ, Hu YL (2010) Theprotective effect of tetramethylpyrazine on cartilage explants and chon-drocytes. J Ethnopharmacol 132:414e420

7. Takasaki J, Kamohara M, Saito T, Matsumoto M, Matsumoto S, Ohishi T, Soga T, Matsushime H, Furuichi K (2001 Sep) Molecular cloning of the platelet P2T(AC) ADP receptor: pharmacological comparison with another ADP receptor, the P2Y(1) receptor. Mol Pharmacol 60(3):432-439 PMID: 11502873 
8. Jacobson KA, Deflorian F, Mishra S, Costanzi S (2011 Sep) Pharmacochemistry of the platelet purinergic receptors. Purinergic Signal 7(3):305-324. doi: 10.1007/s11302-011-9216-0. Epub 2011 Feb 18. PMID: 21484092; PMCID: PMC3166987

9. Lin KH et al (2009) Comparison of the relative activities of inducing platelet apoptosis stimulated by various platelet-activating agents. Platelets 20(8):575-581

10. Leytin V et al (2006) Thrombin-triggered platelet apoptosis. J Thromb Haemost 4(12):2656-2663

11. Wang JJ, Huan SK, Hsieh KH, Chou HC, Hsiao G, Jayakumar T, Sheu JR (2013) Inhibitory effect of midazolam on MMP-9, MMP-1 and MMP-13 expression in PMA-stimulated human chondrocytes via recovery of NF-kB signaling. Arch Med Sci. Apr 20;9(2):332-9. doi: 10.5114/aoms.2012.30949. Epub 2012 Oct 8. PMID: 23671446; PMCID: PMC3648813

12. Gong K, Shao W, Chen H, Wang Z, Luo ZJ (2011) Mar;110(3):145-52 Rat model of lumbar facet joint osteoarthritis associated with facet-mediated mechanical hyperalgesia induced by intra-articular injection of monosodium iodoacetate. J Formos Med Assoc. doi: 10.1016/S0929-6646(11)60024-7. PMID: 21497277

13. Gerwin N, Bendele AM, Glasson S, Carlson CS (2010) The OARSI histopathology initiative recommendations for histological assessments of osteoarthritis in the rat. Osteoarthritis Cartilage. Oct;18 Suppl 3:S24-34. doi: 10.1016/j.joca.2010.05.030. PMID: 20864021

14. Abbracchio MP, Burnstock G, Boeynaems JM, Barnard EA, Boyer JL, Kennedy C, Miras-Portugal MT, King BF, Gachet C, Jacobson KA, Weisman GA (2005 Jan) The recently deorphanized GPR80 (GPR99) proposed to be the P2Y15 receptor is not a genuine P2Y receptor. Trends Pharmacol Sci 26(1):8-9. doi: 10.1016/j.tips.2004.10.010. PMID: 15629198; PMCID: PMC6905457

15. Zhang S, Ye J, Zhang Y, Xu X, Liu J, Zhang SH, Kunapuli SP, Ding Z (2013) Jan;11(1):149-60 P2Y12 protects platelets from apoptosis via PI3k-dependent Bak/Bax inactivation. J Thromb Haemost. doi: 10.1111/jth.12063. PMID: 23140172

16. Haynes SE, Hollopeter G, Yang G, Kurpius D, Dailey ME, Gan WB, Julius D (2006 Dec) The P2Y12 receptor regulates microglial activation by extracellular nucleotides. Nat Neurosci 9(12):1512-1519. doi: 10.1038/nn1805Epub 2006 Nov 19. PMID: 17115040

17. Koizumi S, Shigemoto-Mogami Y, Nasu-Tada K, Shinozaki Y, Ohsawa K, Tsuda M et al (2007) UDP acting at P2Y6 receptors is a mediator of microglial phago-cytosis. Nature 446:1091-1095

18. Preissler J, Grosche A, Lede V, Le Duc D, Krugel K, Matyash V et al (2015) Alteredmicroglial phagocytosis in GPR34-deficient mice. Glia 63:206-215

19. Ashraf S, Mapp PI, Burston J, Bennett AJ, Chapman V, Walsh DA (2014) Augmented pain behavioural responses tointra-articular injection of nerve growth factor in two animalmodels of osteoarthritis. Ann Rheum Dis 73(9):1710-1718

20. Sagar DR, Ashraf S, Xu L et al (2014) Osteoprotegerin reducesthe development of pain behaviour and joint pathology in amodel of osteoarthritis. Ann Rheum Dis 73(8):1558-1565

21. Khan NM, Ahmad I, Haqqi TM (2018) "Nrf2/ARE pathwayattenuates oxidative and apoptotic response in human osteoar-thritis chondrocytes by activating ERK1/2/ELK1-P70S6K-P90RSK 
signaling axis," Free Radical Biology \& Medicine,vol. 116, pp. 159-171,

22. Yuan $Y$, Tan H, Dai $P$ (2017) Krüppel-Like factor 2 regulates degradation of type Il collagen by suppressing the expression of matrix metalloproteinase (MMP)-13. Cell Physiol Biochem 42(6):2159-2168

23. Jiang Y, Hu C, Yu S et al (2015) Cartilage stem/progenitor cells are activated in osteoarthritis via interleukin-1 $\beta /$ nerve growth fac-tor signaling. Arthritis Res Therapy 17(1):327

24. Yeh TT, Wen ZH, Lee HS, Lee $\mathrm{CH}$, Yang Z, Jean YH, Wu SS, Nimni ME, Han B Intra-articular injection of collagenase induced experimental osteoarthritis of the lumbar facet joint in rats. Eur Spine $\mathrm{J}$. 2008May; 17(5):734-42. doi: 10.1007/s00586-008-0594-0. Epub 2008 Jan 26. PMID: 18224353; PMCID: PMC2367420.

25. Gachet $C$ (2005 Nov) The platelet $P 2$ receptors as molecular targets for old and new antiplatelet drugs. Pharmacol Ther 108(2):180-192. doi: 10.1016/j.pharmthera.2005.03.009. Epub 2005 Jun 13. PMID: 15955565

26. Kahner BN, Shankar H, Murugappan S, Prasad GL, Kunapuli SP (2006) Nov;4(11):2317-26 Nucleotide receptor signaling in platelets. J Thromb Haemost. doi: 10.1111/j.1538-7836.2006.02192. x. PMID: 17059469

27. Kauffenstein G, Bergmeier W, Eckly A, Ohlmann P, Léon C, Cazenave JP, Nieswandt B, Gachet C The P2Y (12) receptor induces platelet aggregation through weak activation of the alpha (Ilb)beta (3) integrin--a phosphoinositide 3-kinase-dependent mechanism. FEBS Lett. 2001 Sep 14;505(2):281-90. doi: 10.1016/s0014-5793(01)02824-1. PMID: 11566191

28. Lova P, Paganini S, Hirsch E, Barberis L, Wymann M, Sinigaglia F, Balduini C, Torti M A selective role for phosphatidylinositol 3,4,5-trisphosphate in the Gi-dependent activation of platelet Rap1B. J Biol Chem. 2003 Jan 3;278(1):131-8. doi: 10.1074/jbc.M204821200. Epub 2002 Oct 28. PMID: 12407113

29. Kim S, Jin J, Kunapuli SP (2004) Akt activation in platelets depends on Gi signaling pathways. J Biol Chem. Feb 6;279(6):4186-95. doi: 10.1074/jbc.M306162200. Epub 2003 Nov 17. PMID: 14623889

30. Shankar H, Kahner BN, Prabhakar J, Lakhani P, Kim S, Kunapuli SP G-protein-gated inwardly rectifying potassium channels regulate ADP-induced cPLA2 activity in platelets through Src family kinases. Blood. 2006 Nov 1;108(9):3027-34. doi: 10.1182/blood-2006-03-010330. Epub 2006 Jul 20. PMID: 16857990; PMCID: PMC1895524

31. Gachet C (2001) Dec;74(4):375-81 Identification, characterization, and inhibition of the platelet ADP receptors. Int J Hematol. doi: 10.1007/BF02982079. PMID: 11794691

\section{Figures}

\section{Figure 1}


P2Y12 was elevated in FJOA rat model. A representative image after saffron solid green staining (scale = 100um). B Representative image after H\&E staining (scale $=100 u m$ ). D immunohistochemical method was used to detect the expression of P2Y12, COL2 and MMP13 in rat FJOA and normal cartilage (scale = 100um). E Western blot showed P2Y12 level and COL2 and MMP13 expression in rat FJOA and normal cartilage. Data were presented as mean $\pm \operatorname{SEM}\left(n=3,{ }^{*}<<0.05\right.$, statistically significant difference from non-surgery group)

\section{Figure 2}

Increased P2Y12 expression and apoptosis in SW1353 cells. Western blot analysis showed that after IL$1 \beta(20 \mathrm{ng} / \mathrm{ml})$ stimulation, P2Y12 and apoptosis marker proteins (bax and cleaved caspase-3) were expressed in SW1353 cells. The bar graph B shows the expression ratio of P2Y12, bax and cleaved caspase-3 to GAPDH. * $\mathrm{P}<0.001$ vs. N. C immunofluorescence staining showed that P2Y12 (green) and caspase- 3 lysis (red) were co-localized in SW1353 cells stimulated by IL- $1 \beta .{ }^{*} \mathrm{P}<0.05$ vs. 0 h group.

\section{Figure 3}

Knockdown of P2Y12 promotes IL-1 $\beta$-induced apoptosis in SW1353 cells. A and B demonstrated the effect of silencing P2Y12 by Western blot analysis in SW1353 cells. C knocked P2Y12 with siRNA and treated with IL-1 $\beta$ to increase the level of Caspase3/Bax in SW1353 cells. Bar graph D shows the quantification of the expression ratio of cleaved Caspase-3 to GAPDH. * $\mathrm{P}<0.05$ Vs. untreated cells, * $\mathrm{P}$ $<0.05$ Vs. IL-1 $\beta$-treated non-specific siRNA transfected cells.

\section{Figure 4}

The effect of P2Y12 inhibition on IL-1 3 -induced apoptosis in SW1353 cells. Flow cytometry analysis showed that after IL-1 $\beta$ stimulation, $\mathrm{P} 2 \mathrm{Y} 12$ inhibited the increase of phagocytic membrane protein $\mathrm{v} / \mathrm{pi}$ cells. Bar graph $B$ shows the analysis of apoptosis. ${ }^{*} P<0.05$ Vs. control group, ${ }^{*} P<0.05$ Vs. IL- $1 \beta$ group

\section{Figure 5}

Effects of P2Y12 on IL-1 $\beta$-induced PI3K/AKT signaling pathway in chondrocytes. (A, B) Representative Western blot and quantitative data of P-Akt and Akt in each group. (C-F) P-Akt, Akt, Cleaved Caspase3, Bcl 2. (G) Apoptotic chondrocytes were detected by TUNEL staining. The scale represents $100 u m * p<0.05$ 